\title{
A generalized Pancharatnam geometric phase formula for three-level quantum systems
}

\author{
Arvind $\dagger$, K S Malleshł and N Mukundaई \\ $\dagger$ Department of Physics, Indian Institute of Science, Bangalore 560 012, India \\ $\ddagger$ Department of Studies in Physics, University of Mysore, Mysore 570-996, India \\ $\S$ Center for Theoretical Studies and Department of Physics, Indian Institute of Science, \\ Bangalore 560 012, India
}

\begin{abstract}
We describe a recently developed generalization of the Poincaré sphere method, to represent pure states of a three-level quantum system in a convenient geometrical manner. The construction depends on the properties of the group $S U(3)$ and its generators in the defining representation, and uses geometrical objects and operations in an eight-dimensional real Euclidean space. This construction is then used to develop a generalization of the well known Pancharatnam geometric phase formula, for evolution of a three-level system along a geodesic triangle in state space.
\end{abstract}

\section{Introduction}

Some time after the discovery by Berry in 1984 of the quantum mechanical geometric phase in the framework of cyclic adiabatic evolution [1], Ramaseshan and Nityananda pointed out in an important paper [2] that as early as 1956 Pancharatnam had put forward closely related ideas in the context of polarization optics [3]. Subsequently Berry himself analysed the significance of Pancharatnam's work in the light of later developments [4]. The more recent quantum kinematic approach to the geometric phase clearly brings out the way in which a phase essentially identified by Pancharatnam is one of the two basic ingredients involved in the very definition of the quantum geometric phase, the other being the so-called dynamical phase [5].

Pancharatnam's work made essential use of the Poincaré sphere representation for the manifold of pure polarization states of a plane electromagnetic wave [6]. As is well known, diametrically opposite points on the Poincaré sphere correspond to mutually orthogonal polarization states incapable of interfering with one another. For two states of polarization not mutually orthogonal in this sense Pancharatnam introduced a physically motivated convention or rule which would tell us when these two states are 'in phase', i.e. capable of interfering constructively to the maximum possible extent. More precisely, this relationship is defined at the level of field amplitudes mapping onto given points on the Poincaré sphere. He then went on to show that this relation of being 'in phase' is not transitive. That is, if we take three polarization states $A, B$ and $C$ on the Poincare sphere, and arrange that the fields mapping onto $A$ and $B$ are 'in phase', and similarly those mapping onto $B$ and $C$ are

॥ E-mail address: arvind@physics.iisc.ernet.in

I Also at Jawaharlal Nehru Centre for Advanced Scientific Research, Jakkur Bangalore 560 064, India 
'in phase', then in general the fields mapping onto $A$ and $C$ are not 'in phase'. He also calculated the extent to which these last two fields are 'out of phase' and showed that this 'phase difference' equals one half the solid angle on the Poincare sphere subtended by the spherical triangle $A B C$ obtained by joining the vertices $A, B$ and $C$ by great circle arcs (geodesic arcs) on the sphere.

This fundamental and early result of Pancharatnam has found a natural interpretation in the context of two-level quantum systems, for which the space of pure state density matrices is again the sphere $S^{2}$. In the modern terminology for geometric phases, one is (most often) interested in cyclic evolution in the state space, and the calculation of the associated geometric phase. Evolution along a great circle arc on $S^{2}$ is particularly simple in that it can be generated by a constant (i.e. time-independent) Hamiltonian in such a way that the dynamical phase vanishes. A geodesic triangle on $S^{2}$ is then the simplest and most elementary, yet nontrivial, cyclic evolution one can imagine for a two-level system; it can be produced by a piecewise constant Hamiltonian, and the dynamical phase can be arranged to vanish identically. Then the meaning of Pancharatnam's result is that the resulting geometric phase is one half the solid angle on $S^{2}$ subtended by the triangle [7].

We may note in passing that while Pancharatnam's original result refers principally to the vertices $A, B$ and $C$ of a spherical triangle on $S^{2}$, and calculates the degree of nontransitiveness of the relation of two field amplitudes being 'in phase', in casting it into the modern geometric phase language we are equally concerned with the great circle arcs connecting these vertices, since we deal with continuous cyclic unitary or Hamiltonian evolution of pure quantum states. It is for such evolutions that geometric phases are customarily calculated. Two more remarks are not out of place at this point. One is that in fact geometric phases can be perfectly well defined for noncyclic (and even nonunitary) evolutions, though in this paper we shall not be concerned with them [8]. The other is that for piecewise geodesic and overall cyclic evolutions in a general quantum system, geometric phases get related to certain invariants introduced by Bargmann long ago [9], and these invariants are in conception very close to Pancharatnam's original motivations.

We shall refer to the 'half the solid angle' result as the Pancharatnam formula for geometric phases for two-level systems undergoing piecewise geodesic cyclic evolution along a spherical triangle on $S^{2}$. The main purposes of this paper are: (i) to present a recently developed generalization of the Poincare sphere representation for pure states of three-level quantum systems [10]; and (ii) to then obtain a generalization of the Pancharatnam formula for such systems.

The material of this paper is arranged as follows. Section 2 outlines the generalization of the Poincaré sphere for three-level systems. The Poincaré sphere, $S^{2}$, gets replaced by a certain four-dimensional simply connected region, $\mathcal{O}$, contained wholly within the unit sphere, $S^{7}$, in eight-dimensional real Euclidean space. The transitive action of $S U(3)$ on $\mathcal{O}$, via eight-dimensional orthogonal rotations, some intrinsic properties of $\mathcal{O}$, and a local coordinate system for $\mathcal{O}$, are described so as to assist in forming a picture of this object. Section 3 recalls very briefly the main features of the quantum kinematic approach to the geometric phase of a general quantum system. Both the roles of ray space geodesics and the Bargmann invariants are highlighted. The case of two-level systems, and the statement of the Pancharatnam formula, are then given. It is pointed out that it is a fortunate circumstance that ray space geodesics and geodesics on $S^{2}$ happen to coincide in the case of two-level systems. Finally the general formula for the geometric phase for any cyclic evolution of the three-level system is given. Section 4 discusses the properties of ray space geodesics for three-level systems, and their representation as curves in $\mathcal{O}$. We find that the latter, while they are plane curves, are not geodesics in the geometrical sense on $S^{7}$. We also find that it 
is possible to construct constant Hamiltonians which would give rise to evolution along any such geodesics. Section 5 puts together the ingredients of the previous sections to develop the generalized Pancharatnam formula for three-level systems. This involves describing the most general geodesic triangle for a three-level system state space, and then computing its geometric phase. Whereas a geodesic triangle on $S^{2}$ involves three independent intrinsic parameters, for a geodesic triangle in $\mathcal{O}$ it turns out that four independent intrinsic parameters are needed. The geometric phase then depends on all four of these parameters, and this is borne out by the explicit formula for the phase. Section 6 contains some concluding remarks.

\section{Generalization of the Poincaré sphere representation for three-level systems}

We recall very briefly the salient features of the Poincaré sphere representation for two-level systems, using throughout the notations and terminology of quantum mechanics [12]. We deal with a two-dimensional complex Hilbert space, $\mathcal{H}^{(2)}$, unit vectors in which are denoted by $\psi, \psi^{\prime} \ldots$. The density matrix corresponding to a pure state, $\psi$, is given by the projection

$\rho=\psi \psi^{\dagger}$. Its expansion in terms of the Pauli matrices, $\sigma_{j}$, leads to the Poincare sphere construction:

$$
\begin{aligned}
& \rho=\psi \psi^{\dagger}=\frac{1}{2}(1+\boldsymbol{n} \cdot \boldsymbol{\sigma}) \\
& \rho^{\dagger}=\rho^{2}=\rho \geqslant 0 \quad \operatorname{Tr} \rho=1 \Leftrightarrow \\
& \boldsymbol{n}^{*}=\boldsymbol{n} \quad \boldsymbol{n} \cdot \boldsymbol{n}=1 \Leftrightarrow \boldsymbol{n} \in S^{2} .
\end{aligned}
$$

Thus each pure state in the quantum mechanical sense, or normalized ray, corresponds in a one-to-one manner to a point on the two-dimensional unit sphere, $S^{2}$, embedded in Euclidean three-dimensional space $\mathcal{R}^{3}$. Since

$$
\begin{aligned}
& \rho=\psi \psi^{\dagger}=\frac{1}{2}(1+\boldsymbol{n} \cdot \boldsymbol{\sigma}) \quad \rho^{\prime}=\psi^{\prime} \psi^{\prime \dagger}=\frac{1}{2}\left(1+\boldsymbol{n}^{\prime} \cdot \boldsymbol{\sigma}\right) \Rightarrow \\
& \operatorname{Tr}\left(\rho^{\prime} \rho\right)=\left|\left(\psi^{\prime}, \psi\right)\right|^{2}=\frac{1}{2}\left(1+\boldsymbol{n}^{\prime} \cdot \boldsymbol{n}\right)
\end{aligned}
$$

we see that diametrically opposite points on $S^{2}$ correspond to mutually orthogonal rays or Hilbert space vectors. Here $\left(\psi^{\prime}, \psi\right)$ is the inner product in $\mathcal{H}^{(2)}$. Finally, if a vector, $\psi \in \mathcal{H}^{(2)}$, is subjected to a transformation, $u \in S U(2)$, the representative point, $\boldsymbol{n} \in S^{2}$ undergoes an orthogonal rotation belonging to $S O(3)$ :

$$
\begin{aligned}
& \psi^{\prime}=u \psi \quad u \in S U(2) \Rightarrow \\
& n_{j}^{\prime}=R_{j k}(u) n_{k} \\
& R_{j k}(u)=\frac{1}{2} \operatorname{Tr}\left(\sigma_{j} u \sigma_{k} u^{\dagger}\right) \quad R(u) \in S O(3) .
\end{aligned}
$$

As is well known, all elements $\mathcal{R} \in S O(3)$ are realized in this way, and we have the coset space identifications $S^{2}=S U(2) / U(1)=S O(3) / S O(2)$.

Now we present the natural generalization of this construction to three-level systems. We deal with a three-dimensional complex Hilbert space, $\mathcal{H}^{(3)}$, elements of which will be again denoted by $\psi, \psi^{\prime}, \ldots$ The roles of $S U(2)$ and the Pauli matrices, $\sigma_{j}$, are now played by the group $S U(3)$ via its defining representation, and the eight Hermitian generators, $\lambda_{r}$, in this representation [13]:

$$
S U(3)=\left\{A=3 \times 3 \text { complex matrix } \mid A^{\dagger} A=1, \operatorname{det} A=1\right\}
$$




$$
\begin{array}{rlrl}
\lambda_{1} & =\left(\begin{array}{lll}
0 & 1 & 0 \\
1 & 0 & 0 \\
0 & 0 & 0
\end{array}\right) & \lambda_{2}=\left(\begin{array}{ccc}
0 & -\mathrm{i} & 0 \\
\mathrm{i} & 0 & 0 \\
0 & 0 & 0
\end{array}\right) \\
\lambda_{3}=\left(\begin{array}{ccc}
1 & 0 & 0 \\
0 & -1 & 0 \\
0 & 0 & 0
\end{array}\right) & \lambda_{4}=\left(\begin{array}{ccc}
0 & 0 & 1 \\
0 & 0 & 0 \\
1 & 0 & 0
\end{array}\right) \\
\lambda_{5}=\left(\begin{array}{ccc}
0 & 0 & -\mathrm{i} \\
0 & 0 & 0 \\
\mathrm{i} & 0 & 0
\end{array}\right) & \lambda_{6}=\left(\begin{array}{ccc}
0 & 0 & 0 \\
0 & 0 & 1 \\
0 & 1 & 0
\end{array}\right) \\
\lambda_{7}=\left(\begin{array}{ccc}
0 & 0 & 0 \\
0 & 0 & -\mathrm{i} \\
0 & \mathrm{i} & 0
\end{array}\right) & \lambda_{8}=\frac{1}{\sqrt{3}}\left(\begin{array}{ccc}
1 & 0 & 0 \\
0 & 1 & 0 \\
0 & 0 & -2
\end{array}\right) .
\end{array}
$$

The matrices $\lambda_{r}$ obey characteristic commutation and anticommutation relations:

$$
\begin{aligned}
& {\left[\lambda_{r}, \lambda_{s}\right]=2 \mathrm{i} f_{r s t} \lambda_{t} \quad\left\{\lambda_{r}, \lambda_{s}\right\}=\frac{4}{3} \delta_{r s}+2 d_{r s t} \lambda_{t}} \\
& f_{123}=1 \quad f_{458}=f_{678}=\frac{\sqrt{3}}{2} \quad f_{147}=f_{246}=f_{257}=f_{345}=f_{516}=f_{637}=\frac{1}{2} \\
& d_{118}=d_{228}=d_{338}=-d_{888}=\frac{1}{\sqrt{3}} \quad d_{448}=d_{558}=d_{668}=d_{778}=-\frac{1}{2 \sqrt{3}} \\
& d_{146}=d_{157}=-d_{247}=d_{256}=d_{344}=d_{355}=-d_{366}=-d_{377}=\frac{1}{2} .
\end{aligned}
$$

Here we have given the independent nonvanishing components of the completely antisymmetric $f_{r s t}$ and the completely symmetric $d_{r s t}$; the former are the $S U$ (3) structure constants. These $f$ and $d$ symbols allow us to define both antisymmetric and symmetric products among real vectors $\boldsymbol{a}, \boldsymbol{b}, \ldots$ in real eight-dimensional Euclidean space, $\mathcal{R}^{8}$, the result in each case being another such vector [10]:

$$
\begin{array}{lr}
\left(\boldsymbol{a}_{\wedge} \boldsymbol{b}\right)_{r}=f_{r s t} a_{s} b_{t} & \boldsymbol{a}_{\wedge} \boldsymbol{b}=-\boldsymbol{b}_{\wedge} \boldsymbol{a} \\
(\boldsymbol{a} \star \boldsymbol{b})_{r}=\sqrt{3} d_{r s t} a_{s} b_{t} & \boldsymbol{a} \star \boldsymbol{b}=\boldsymbol{b} \star \boldsymbol{a} .
\end{array}
$$

The significance of these definitions is that $\boldsymbol{a}_{\wedge} \boldsymbol{b}$ and $\boldsymbol{a} \star \boldsymbol{b}$ transform just as $\boldsymbol{a}$ and $\boldsymbol{b}$ do, under the eight-dimensional adjoint representation of $S U(3)$. The matrices of this representation are defined similarly to equation (2.3):

$$
\begin{aligned}
& A \in S U(3) \rightarrow D_{r s}(A)=\frac{1}{2} \operatorname{Tr}\left(\lambda_{r} A \lambda_{s} A^{\dagger}\right) \\
& D\left(A^{\prime}\right) D(A)=D\left(A^{\prime} A\right) \\
& D(A) \in S O(8) .
\end{aligned}
$$

However, in contrast to the $S U(2)-S O(3)$ case, here the matrices $D(A)$ that arise are only an eight-parameter family, and so a very small portion indeed of the full twenty-eightparameter group $S O(8)$. In any case the required properties of the products (2.6) are:

$$
\begin{aligned}
& D(A) \boldsymbol{a}_{\wedge} D(A) \boldsymbol{b}=D(A)\left(\boldsymbol{a}_{\wedge} \boldsymbol{b}\right) \\
& D(A) \boldsymbol{a} \star D(A) \boldsymbol{b}=D(A)(\boldsymbol{a} \star \boldsymbol{b}) .
\end{aligned}
$$

With this background, we can handle general pure state density matrices for three-level systems [10]. Given a normalized $\psi \in \mathcal{H}^{(3)}$, we form the density matrix $\rho=\psi \psi^{\dagger}$ and expand it in terms of the unit matrix and the $\lambda_{r}$ :

$$
\begin{aligned}
& \psi \in \mathcal{H}^{3} \quad(\psi, \psi)=1 \\
& \rho=\psi \psi^{\dagger}=\frac{1}{3}(1+\sqrt{3} \boldsymbol{n} \cdot \boldsymbol{\lambda}) .
\end{aligned}
$$

We then find in place of equation (2.2):

$$
\rho^{\dagger}=\rho^{2}=\rho \geqslant 0 \quad \operatorname{Tr} \rho=1 \Leftrightarrow \boldsymbol{n}^{\star}=\boldsymbol{n} \quad \boldsymbol{n} \cdot \boldsymbol{n}=1 \quad \boldsymbol{n} \star \boldsymbol{n}=\boldsymbol{n} .
$$


Thus each normalized ray for the three-level system corresponds uniquely in a one-to-one manner to a unit vector, $\boldsymbol{n} \in S^{7}$, the seven-dimensional unit sphere in $\mathcal{R}^{8}$, which moreover obeys the condition $\boldsymbol{n} \star \boldsymbol{n}=\boldsymbol{n}$. The set of all such real unit vectors in $\mathcal{R}^{8}$, a subset of $S^{7}$, is the analogue of the Poincaré sphere for three-level systems. Since it is in fact a very small part of $S^{7}$, we give it a special symbol:

$$
\mathcal{O}=\left\{\boldsymbol{n} \in \mathcal{R}^{8} \mid \boldsymbol{n} \cdot \boldsymbol{n}=1, \boldsymbol{n} \star \boldsymbol{n}=\boldsymbol{n}\right\} \subset S^{7} \subset \mathcal{R}^{8} .
$$

This set, $\mathcal{O}$, is a connected, simply connected four-dimensional region contained in $S^{7}$, and its points correspond one-to-one to pure states of a three-level system. It is in fact a representation of the coset space $S U(3) / U(2)$.

Some interesting geometric properties of $\mathcal{O}$ may be mentioned. For two unit vectors, $\psi$ and $\psi^{\prime} \in \mathcal{H}^{(3)}$, we find:

$$
\begin{aligned}
& \rho=\psi \psi^{\dagger} \quad \rho^{\prime}=\psi^{\prime} \psi^{\prime \dagger} \Rightarrow \operatorname{Tr}\left(\rho^{\prime} \rho\right)=\left|\left(\psi^{\prime}, \psi\right)\right|^{2}=\frac{1}{3}\left(1+2 \boldsymbol{n}^{\prime} \cdot \boldsymbol{n}\right) \\
& 0 \leqslant \operatorname{Tr}\left(\rho^{\prime} \rho\right) \leqslant 1 \Leftrightarrow 0 \leqslant \cos ^{-1}\left(\boldsymbol{n}^{\prime} \cdot \boldsymbol{n}\right) \leqslant \frac{2 \pi}{3} .
\end{aligned}
$$

Thus mutually orthogonal vectors in $\mathcal{H}^{(3)}$ do not lead to antipodal or diametrically opposite points on $\mathcal{O}$, but rather to points with a maximum opening angle of $\frac{2 \pi}{3}$ radians. Indeed, if $\boldsymbol{n} \in \mathcal{O}$, then $-\boldsymbol{n} \notin \mathcal{O}$. If one takes the three canonical basis vectors of $\mathcal{H}^{(3)}$ as usual, they lead to three distinguished points or 'poles' on $\mathcal{O}$ :

$$
\begin{array}{lcr}
(1,0,0)^{T} \rightarrow n_{3}=\frac{\sqrt{3}}{2} & n_{8}=\frac{1}{2} & \text { rest zero } \\
(0,1,0)^{T} \rightarrow n_{3}=-\frac{\sqrt{3}}{2} & n_{8}=\frac{1}{2} & \text { rest zero } \\
(0,0,1)^{T} \rightarrow n_{8}=-1 & \text { rest zero } &
\end{array}
$$

each making an angle of $\frac{2 \pi}{3}$ with any other. These properties of $\mathcal{O}$ may help one make some sort of mental picture of this geometrical object embedded in $S^{7}$.

The action of $S U(3)$ on vectors in $\mathcal{H}^{(3)}$ leads to adjoint action on $\mathcal{O}$ :

$$
A \in S U(3): \psi^{\prime}=A \psi \Rightarrow \boldsymbol{n}^{\prime}=D(A) \boldsymbol{n} .
$$

Thus one has here a (small set of) rigid eight-dimensional orthogonal rotations, which will prove convenient later on. Moreover, since $\mathcal{O}$ is the coset space $S U(3) / U(2)$, this adjoint action of $S U$ (3) on $\mathcal{O}$ is transitive. This will also be exploited later. General $S O(8)$ rotations of course do not preserve the region $\mathcal{O}$ of $S^{7}$.

For practical calculations it is convenient to introduce four independent local angle type variables which can be used as coordinates over (almost all of) $\mathcal{O}$. Let us write a general unit vector, $\psi \in \mathcal{H}^{(3)}$, as

$$
\psi=\left(\begin{array}{l}
\psi_{1} \\
\psi_{2} \\
\psi_{3}
\end{array}\right) \quad \psi^{\dagger} \psi=\left|\psi_{1}\right|^{2}+\left|\psi_{2}\right|^{2}+\left|\psi_{3}\right|^{2}=1 .
$$

Then omitting the part of $\mathcal{O}$ corresponding to $\psi_{3}=0$ (this is a two-dimensional region, essentially an $S^{2}$, see below), over the rest of $\mathcal{O}$ we introduce $\theta, \phi$ and $\chi_{1}, \chi_{2}$ in this wayt:

$$
\begin{aligned}
& \left(\psi_{1}, \psi_{2}, \psi_{3}\right)=(\text { overall phase })\left(\mathrm{e}^{\mathrm{i} \chi_{1}} \sin \theta \cos \phi, \mathrm{e}^{\mathrm{i} \chi_{2}} \sin \theta \sin \phi, \cos \theta\right) \\
& 0 \leqslant \theta<\frac{\pi}{2} \quad 0 \leqslant \phi \leqslant \frac{\pi}{2} \quad 0 \leqslant \chi_{1}, \chi_{2}<2 \pi .
\end{aligned}
$$

$\dagger$ The choice of these variables differs from that in [10]. 


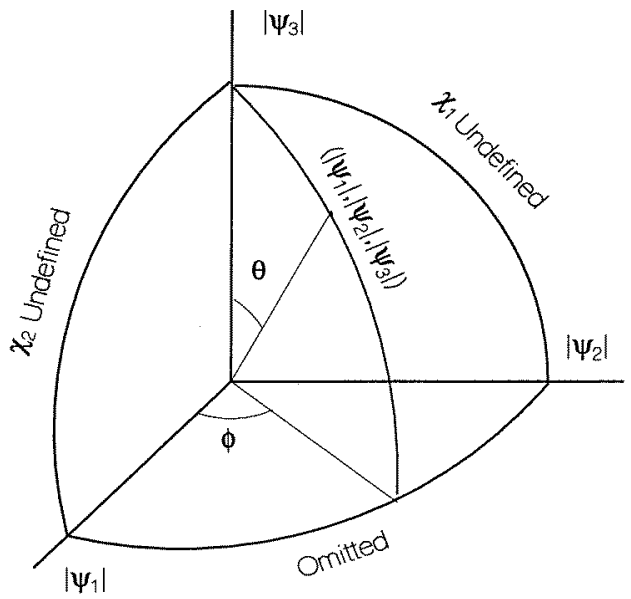

Figure 1. A pictorial description of almost all of the space $\mathcal{O}$ (the space of all states of the three-level system). The local coordinates $\theta, \phi$ and $\chi_{1}, \chi_{2}$ are such that $\theta$ and $\phi$ define a point in the positive octant of $S^{2}$, i.e. $0 \leqslant \theta, \phi \leqslant \frac{\pi}{2}$ and for a given point on this octant we have a torus defined by two-angle variables $0 \leqslant \chi_{1}, \chi_{2}<2 \pi$.

The limits on $\theta, \phi$ reflect the nonvanishing of $\psi_{3}$, and the fact that the real three-dimensional unit vector $\left(\left|\psi_{1}\right|,\left|\psi_{2}\right|,\left|\psi_{3}\right|\right)$ has non-negative components. Thus $\theta, \phi$ denotes a point on the first octant of an $S^{2}$. Given $\left|\psi_{3}\right|>0, \chi_{1}$ is the phase of $\psi_{1}$ relative to $\psi_{3}$ (and is well defined except when $\phi=\frac{\pi}{2}$ ); and $\chi_{2}$ is the phase of $\psi_{2}$ relative to $\psi_{3}$ (and is well defined except when $\phi=0$ ), see figure 1 . We need to remember that $\theta, \phi$ determine the magnitudes of the components of $\psi$, while $\chi_{1}, \chi_{2}$ give their relative phases. All four taken together determine one point in the portion of $\mathcal{O}$ with $\psi_{3} \neq 0$.

We can easily obtain the expressions for $n_{r}$ in these local coordinates. Combining equations (2.9) and (2.16) we get:

$n_{r}=\frac{\sqrt{3}}{2} \psi^{\dagger} \lambda_{r} \psi$

$\boldsymbol{n}=\sqrt{3}\left(\sin ^{2} \theta \sin \phi \cos \phi \cos \left(\chi_{2}-\chi_{1}\right), \sin ^{2} \theta \sin \phi \cos \phi \sin \left(\chi_{2}-\chi_{1}\right)\right.$,

$$
\begin{aligned}
& \frac{1}{2} \sin ^{2} \theta\left(\cos ^{2} \phi-\sin ^{2} \phi\right), \sin \theta \cos \theta \cos \phi \cos \chi_{1}, \\
& -\sin \theta \cos \theta \cos \phi \sin \chi_{1}, \sin \theta \cos \theta \sin \phi \cos \chi_{2}, \\
& \left.-\sin \theta \cos \theta \sin \phi \sin \chi_{2}, \frac{1}{2 \sqrt{3}}\left(1-3 \cos ^{2} \theta\right)\right) .
\end{aligned}
$$

The recovery of the Poincare sphere, $S^{2}$, for a two-dimensional subspace of $\mathcal{H}^{(3)}$ is straightforward. Consider, as an example, vectors $\psi \in \mathcal{H}^{(3)}$ with a vanishing third component (just the points of $\mathcal{O}$ omitted in the local coordinatization $(2.16)$ of $\mathcal{O}$ ):

$$
\begin{aligned}
& \psi=\left(\begin{array}{c}
\psi_{1} \\
\psi_{2} \\
0
\end{array}\right)=\left(\begin{array}{c}
\mathrm{e}^{\mathrm{i} \chi_{1}} \cos \phi \\
\mathrm{e}^{\mathrm{i} \chi_{2}} \sin \phi \\
0
\end{array}\right) \\
& 0 \leqslant \phi \leqslant \frac{\pi}{2} \quad 0 \leqslant \chi_{1}, \quad \chi_{2}<2 \pi .
\end{aligned}
$$


Then the eight-vector $\boldsymbol{n}$ has only four nonvanishing components:

$$
\begin{aligned}
& \left(n_{1}, n_{2}, n_{3}\right)=\frac{\sqrt{3}}{2}\left(\sin 2 \phi \cos \left(\chi_{2}-\chi_{1}\right), \sin 2 \phi \sin \left(\chi_{2}-\chi_{1}\right), \cos 2 \phi\right) \\
& n_{8}=\frac{1}{2} \\
& n_{4}=n_{5}=n_{6}=n_{7}=0 .
\end{aligned}
$$

As $\phi$ and $\left(\chi_{2}-\chi_{1}\right)$ vary in the appropriate ranges, we see that we obtain a certain sphere, $S^{2}$, embedded within $\mathcal{O}$, centred on the point $\left(0,0,0,0,0,0,0, \frac{1}{2}\right)$, of radius $\frac{\sqrt{3}}{2}$, and contained entirely within the $1-2-3-8$ subspace of $\mathcal{R}^{8}$. If we consider two-dimensional subspaces in $\mathcal{H}^{(3)}$ different from (2.18), we clearly obtain $S U$ (3) transforms of the above situation. All these various $S^{2}$,s are off-centre in $\mathcal{R}^{8}$ : indeed their centres lie on a sphere in $\mathcal{R}^{8}$ centred at the origin of $\mathcal{R}^{8}$ and of radius $\frac{1}{2}$.

\section{Background to the geometric phase and Pancharatnam's formula}

Consider a general quantum mechanical system whose pure states are described by unit vectors in a complex Hilbert space, $\mathcal{H}$, of any dimension. The corresponding ray space will be denoted by $\mathcal{R}$. Let $\mathcal{C}$ be a continuous piecewise smooth parametrized curve of unit vectors in $\mathcal{H}$ :

$$
\mathcal{C}=\left\{\psi(s) \mid s_{1} \leqslant s \leqslant s_{2}\right\} \subset \mathcal{H}
$$

and let $C$ be its image in $\mathcal{R}$, likewise continuous and piecewise smooth:

$$
C=\left\{\rho(s)=\psi(s) \psi(s)^{\dagger} \mid s_{1} \leqslant s \leqslant s_{2}\right\} \subset \mathcal{R} .
$$

In case $\psi\left(s_{2}\right)$ and $\psi\left(s_{1}\right)$ determine the same ray, and in particular $\psi\left(s_{2}\right)=\psi\left(s_{1}\right)$ in which case $\mathcal{C}$ is closed, the image $C$ is closed; however, in general we need not assume this. The geometric phase associated with $C$ is the difference between a total (or Pancharatnam) phase and a dynamical phase, each of which is a functional of $\mathcal{C}$ [5]:

$$
\begin{aligned}
& \varphi_{g}[C]=\varphi_{p}[\mathcal{C}]-\varphi_{\text {dyn }}[\mathcal{C}] \\
& \varphi_{p}[\mathcal{C}]=\arg \left(\psi\left(s_{1}\right), \psi\left(s_{2}\right)\right) \\
& \varphi_{\text {dyn }}[\mathcal{C}]=\operatorname{Im} \int_{s_{1}}^{s_{2}} \mathrm{~d} s(\psi(s), \dot{\psi}(s)) .
\end{aligned}
$$

The quantity, $\varphi_{g}[C]$, is invariant under both local smooth phase changes in $\psi(s)$, and under smooth reparametrizations - for these reasons it is a geometric quantity dependent on $C$ rather than on $\mathcal{C}$.

In this context an important role is played by geodesics in the space $\mathcal{R}$ [5]. Given the continuous curve $C \subset \mathcal{R}$ (with nonorthogonal end points for definiteness), a nondegenerate positive definite length functional, $\mathcal{L}[C]$, can be set up, which is also reparametrization invariant:

$$
\mathcal{L}[C]=\int_{s_{1}}^{s_{2}} \mathrm{~d} s\{(\dot{\psi}(s), \dot{\psi}(s))-(\psi(s), \dot{\psi}(s))(\dot{\psi}(s), \psi(s))\}^{\frac{1}{2}} .
$$

Extremizing this functional (with fixed end points), we arrive at the concept of geodesics in $\mathcal{R}$. Any Hilbert space lift of such a geodesic, with any choice of parametrization, may then be called a geodesic in $\mathcal{H}$. It then turns out that every geodesic in $\mathcal{R}$ has vanishing geometric phase:

$$
C=\text { geodesic in } \mathcal{R} \Rightarrow \varphi_{g}[C]=0
$$


and this accounts for their importance. The simplest description of a geodesic, which can always be achieved, is as follows. Let the end points of $C$ be $\rho^{(1)}$ and $\rho^{(2)}$, assumed nonorthogonal, and choose unit vectors $\psi^{(1)}$ and $\psi^{(2)}$ such that:

$$
\begin{aligned}
& \rho^{(1)}=\psi^{(1)} \psi^{(1) \dagger} \quad \rho^{(2)}=\psi^{(2)} \psi^{(2) \dagger} \\
& \left(\psi^{(1)}, \psi^{(2)}\right)=\text { real positive. }
\end{aligned}
$$

Thus $\psi^{(1)}$ and $\psi^{(2)}$ are 'in phase' in the Pancharatnam sense. Then the geodesic $G_{\text {geo }} \subset \mathcal{R}$ connecting $\rho^{(1)}$ to $\rho^{(2)}$ is the ray space image of the following curve $\mathcal{C}_{\text {geo }} \subset \mathcal{H}$ :

$\mathcal{C}_{\text {geo }}=\left\{\psi(s) \mid 0 \leqslant s \leqslant s_{0}\right\}$

$\psi(s)=\psi(0) \cos s+\dot{\psi}(0) \sin s$

$\psi(0)=\psi^{(1)} \quad \dot{\psi}(0)=\left(\psi^{(2)}-\psi^{(1)}\left(\psi^{(1)}, \psi^{(2)}\right)\right) /\left(1-\left(\psi^{(1)}, \psi^{(2)}\right)^{2}\right)^{\frac{1}{2}}$

$s_{0}=\cos ^{-1}\left(\psi^{(-1)}, \psi^{(2)}\right)$.

Exploiting the fundamental result (3.5) we obtain a very attractive expression for the geometric phase in the following particular situation. Choose a set of points $\rho^{(1)}, \rho^{(2)}, \ldots, \rho^{(n)} \in \mathcal{R}$ in a definite sequence, assume for definiteness that no two consecutive points are mutually orthogonal, and also that $\rho^{(n)}$ and $\rho^{(1)}$ are nonorthogonal. Connect $\rho^{(1)}$ to $\rho^{(2)}, \rho^{(2)}$ to $\rho^{(3)}, \ldots, \rho^{(n)}$ to $\rho^{(1)}$ by geodesic arcs, so that we obtain a closed curve $C \subset \mathcal{R}$ in the form of an $n$-sided polygon made up of geodesic pieces. Then we have [5]:

$$
\begin{aligned}
& C=\text { geodesic polygon in } \mathcal{R} \text { with vertices } \rho^{(1)}, \rho^{(2)}, \ldots, \rho^{(n)} \\
& \begin{aligned}
\varphi_{g}[C]=-\arg \left(\psi^{(1)}, \psi^{(2)}\right)\left(\psi^{(2)}, \psi^{(3)}\right) \cdots\left(\psi^{(n)}, \psi^{(1)}\right) \\
\quad=-\arg \operatorname{Tr}\left(\rho^{(1)} \rho^{(2)} \ldots \rho^{(n)}\right)
\end{aligned} \\
& \begin{array}{c}
\rho^{(1)}=\psi^{(1)} \psi^{(1) \dagger} \quad \rho^{(2)}=\psi^{(2)} \psi^{(2) \dagger}, \ldots \quad \rho^{(n)}=\psi^{(n)} \psi^{(n) \dagger} .
\end{array}
\end{aligned}
$$

Here it is evident that the phases of the vectors $\psi^{(1)}, \psi^{(2)}, \ldots, \psi^{(n)}$ can be freely chosen. The result (3.8) connects the geometric phase for a closed polygon to the Bargmann invariant of quantum mechanics, the expression $\left(\psi^{(1)}, \psi^{(2)}\right)\left(\psi^{(2)}, \psi^{(3)}\right) \ldots\left(\psi^{(n)}, \psi^{(1)}\right)$, namely: the former is the negative of the argument of the latter. The point to emphasize is that the definition of the Bargmann invariant requires specifying just the vertices of the polygon, while the definition of the geometric phase requires also connecting them in sequence by geodesic arcs so that we have a closed loop $C \subset \mathcal{R}$.

With this background from the general theory of the geometric phase we relate these results to the case of two-level systems, quote the Pancharatnam formula and then give the general expression for $\varphi_{g}[C]$ for three-level systems. For two-level systems we have seen that the space $\mathcal{R}$ is the Poincare sphere $S^{2}$. It is now a happy coincidence that geodesics in $\mathcal{R}$ map exactly on to geodesics on $S^{2}$ in the more familiar Euclidean sense. This can be seen as follows. Without loss of generality, by using a suitable $S U(2)$ transformation, we may assume that the points $\rho^{(1)}$ and $\rho^{(2)}$ to be connected by a geodesic are the points $\boldsymbol{n}^{(1)}=(0,0,1), \boldsymbol{n}^{(2)}=(\sin 2 \alpha, 0, \cos 2 \alpha)$ on $S^{2}$, with representative vectors $\psi^{(1)}$ and $\psi^{(2)} \in \mathcal{H}^{(2)}$ chosen as follows:

$$
\psi^{(1)}=\left(\begin{array}{l}
1 \\
0
\end{array}\right) \quad \psi^{(2)}=\left(\begin{array}{c}
\cos \alpha \\
\sin \alpha
\end{array}\right) .
$$


Then applying the result (3.7), the ray space geodesic connecting $\rho^{(1)}$ to $\rho^{(2)}$ is determined as follows:

$$
\begin{aligned}
\psi(s) & =\left(\begin{array}{c}
\cos s \\
\sin s
\end{array}\right) \\
\boldsymbol{n}(s) & =\operatorname{Tr} \rho(s) \boldsymbol{\sigma}=(\psi(s), \boldsymbol{\sigma} \psi(s)) \\
& =(\sin 2 s, 0, \cos 2 s) \quad 0 \leqslant s \leqslant \alpha .
\end{aligned}
$$

We see that the curve described by $\boldsymbol{n}(s)$ on $S^{2}$ is indeed a great circle arc, a part of the 'Greenwich meridian'; and by the action of $S U(2)$ on $\mathcal{R}$ translated into the action of $S O$ (3) on $S^{2}$, we conclude that any ray space geodesic in $\mathcal{R}$ appears as some great circle arc on $S^{2}$. Thus the two definitions of geodesics do coincide in this case.

Let now $A, B$ and $C$ be any three points on $S^{2}$, no two being diametrically opposite to one another. Joining them by great circle arcs (each less than $\pi$ in extent) we get a geodesic triangle $\triangle(A, B, C)$ on $S^{2}$. Then the Pancharatnam formula $[3,4,2]$ is the statement that for any two-level system:

$\varphi_{g}[\triangle(A, B, C)]=\frac{1}{2} \Omega$

$\Omega=$ solid angle subtended by the triangle $A, B, C$ at the origin of $S^{2}$.

Here the right-hand side is interpreted to be positive (negative) if, as viewed from the outside of $S^{2}$, the triangle $A B C$ is described in the counter clockwise (clockwise) sense. It is this formula that we shall generalize in section 5 .

Now we give the general formula for geometric phases for three-level systems [10]. Consider a closed loop $C \subset \mathcal{O}$, and assume for definiteness that $\psi_{3} \neq 0$ throughout. Then $\varphi_{g}[C]$ is given by the following integral along $C$ :

$$
\varphi_{g}[C]=-\oint_{C \subset \mathcal{O}} \sin ^{2} \theta\left(\cos ^{2} \phi \mathrm{d} \chi_{1}+\sin ^{2} \phi \mathrm{d} \chi_{2}\right) .
$$

We note that this formula holds when $C$ is a closed loop. In the succeeding sections we develop the properties of geodesics in $\mathcal{O}$ and then generalize equation (3.11).

\section{Ray space geodesics for three-level systems}

We have seen that the transitive action of $S U(3)$ on the ray space, $\mathcal{O}$, for three-level systems is given by (an eight-parameter subset of) rigid orthogonal rotations in real Euclidean eightdimensional space, when points of $\mathcal{O}$ are identified with vectors $\boldsymbol{n}$ as in equation (2.11). Based on this we may describe the details of any one conveniently chosen geodesic in $\mathcal{O}$; and then any other would be a suitable $S O(8)$ transform of this one, so that the geometrical shape and structure in an intrinsic sense are unaltered.

Guided by the constructions of section 3, equations (3.9) and (3.10) let us choose two points in $\mathcal{O}$ corresponding to the following two unit vectors in $\mathcal{H}^{(3)}$ :

$$
\begin{aligned}
& \psi^{(1)}=\left(\begin{array}{l}
0 \\
0 \\
1
\end{array}\right) \quad \psi^{(2)}=\left(\begin{array}{c}
0 \\
\sin \alpha \\
\cos \alpha
\end{array}\right) \\
& \boldsymbol{n}^{(1)}=(0,0,0,0,0,0,0,-1) \\
& \boldsymbol{n}^{(2)}=\frac{\sqrt{3}}{2}\left(0,0,-\sin ^{2} \alpha, 0,0,2 \sin \alpha \cos \alpha, 0, \frac{1}{\sqrt{3}}\left(1-3 \cos ^{2} \alpha\right)\right) .
\end{aligned}
$$

It is easy to see that, given any pair of three-level pure-state density matrices, $\rho^{(1)}$ and $\rho^{(2)}$, such that $\operatorname{Tr}\left(\rho^{(1)} \rho^{(2)}\right)=\cos ^{2} \alpha>0$, we can exploit the action of $S U(3)$ on $\mathcal{H}^{(3)}$, and 
freedom of phases, to put $\rho^{(1)}$ and $\rho^{(2)}$ into the configurations corresponding to the vectors $\psi^{(1)}$ and $\psi^{(2)}$ above. Then the ray space geodesic $C_{\text {geo }}^{(0)}$ connecting $\boldsymbol{n}^{(1)}$ and $\boldsymbol{n}^{(2)}$ is easily found on the basis of the general formula (3.7):

$$
\begin{aligned}
\psi(s)=\left(\begin{array}{c}
0 \\
\sin s \\
\cos s
\end{array}\right) & \\
C_{\mathrm{geo}}^{(0)}: \boldsymbol{n}(s) & =\frac{\sqrt{3}}{2} \psi(s)^{\dagger} \lambda \psi(s) \\
& =\frac{\sqrt{3}}{2}\left(0,0,-\sin ^{2} s, 0,0,2 \sin s \cos s, 0, \frac{1}{\sqrt{3}}\left(1-3 \cos ^{2} s\right)\right) \quad 0 \leqslant s \leqslant \alpha .
\end{aligned}
$$

This curve $\{\boldsymbol{n}(s)\} \subset \mathcal{O}$ has only three nonvanishing components, namely, $n_{3}(s), n_{6}(s)$ and $n_{8}(s)$. The interesting questions are whether it is a plane curve, whether it coincides with a geodesic arc as defined in the sense of eight-dimensional Euclidean geometry on $S^{7}$, and whether it has any other important geometric features.

The first observation we may make is that since the components $n_{1}, n_{2}, n_{4}, n_{5}$ and $n_{7}$ all vanish, this curve, $C_{\text {geo }}^{(0)}$, lies entirely in a three-dimensional subspace of $\mathcal{R}^{8}$. By $S U(3)$ action this statement is then true for all ray space geodesics $C_{\text {geo }}$ when drawn in $\mathcal{O}$. Next we remark that a geodesic on $S^{7}$ in the Euclidean sense would be part of the intersection of a two-dimensional plane in $\mathcal{R}^{8}$ passing through the origin, with $S^{7}$. We can immediately see that $C_{\text {geo }}^{(0)}$ is not of this kind; for example, the three vectors $\boldsymbol{n}(0), \boldsymbol{n}\left(\frac{\alpha}{2}\right)$ and $\boldsymbol{n}(\alpha)$ are easily checked to be linearly independent. Thus, in striking contrast to the situation on the Poincaré sphere, $S^{2}$, for two-level systems, here ray space geodesics on $\mathcal{O}$ are not geodesic arcs in the sense of Euclidean eight-dimensional geometry.

Nevertheless we can show that $C_{\text {geo }}^{(0)}$ is a plane curve, though the plane on which it lies is off centre in $\mathcal{R}^{8}$, i.e. it does not pass through the origin or the centre of $S^{7}$. For this we need to work with the combinations $\frac{\sqrt{3} n_{3}+n_{8}}{2}$ and $\frac{n_{3}-\sqrt{3} n_{8}}{2}$ obtained from $n_{3}$ and $n_{8}$ by an orthogonal transformation. Then we find:

$$
\begin{aligned}
& \frac{\sqrt{3} n_{3}(s)+n_{8}(s)}{2}=-\frac{1}{2} \\
& n_{6}(s)=\sqrt{3} \sin s \cos s \\
& \frac{n_{3}(s)-\sqrt{3} n_{8}(s)}{2}=-\frac{\sqrt{3}}{2}\left(1-2 \cos ^{2} s\right) .
\end{aligned}
$$

Thus, in the three-dimensional $n_{3}-n_{6}-n_{8}$ subspace, this geodesic $C_{\text {geo }}^{(0)}$ is a curve lying in the two-dimensional plane $\frac{\sqrt{3} n_{3}+n_{8}}{2}=-\frac{1}{2}$. Ray space geodesics $C_{\text {geo }}$ for three-level systems, when described as curves $\{\boldsymbol{n}(s)\} \subset \mathcal{O}$, are really like arcs of constant latitude on $S^{2}$, and not geodesic arcs at all. For this reason, hereafter the term geodesic will refer exclusively to ray space geodesics, with no further qualification.

In the description of $\mathcal{O}$ using coordinates $\theta, \phi$ and $\chi_{1}, \chi_{2}$ the geodesic $C_{\text {geo }}^{(0)}$ appears as follows:

$C_{\mathrm{geo}}^{(0)}: \theta(s)=s \quad \phi(s)=\frac{\pi}{2} \quad \chi_{1}(s)$ undefined $\quad \chi_{2}(s)=0 \quad 0 \leqslant s \leqslant \alpha$.

Thus the projection of $C_{\text {geo }}^{(0)}$ onto the octant of $S^{2}$ with spherical polar angles $\theta, \phi$ happens to be a great circle arc in the usual sense. However, this is not expected to be true for a general geodesic $C_{\text {geo }} \subset \mathcal{O}$. 
One can now ask for the most general three-level system Hamiltonian which reproduces the evolution of $\psi(s)$ with respect to $s$, as already specified in equation (4.2), and whether it can be independent of $s$. A general Hermitian Hamiltonian can be written in terms of nine real functions of $s$ as

$$
H(s)=h_{0}(s)+\boldsymbol{h}(s) \cdot \boldsymbol{\lambda}
$$

while the quantum mechanical evolution equations for $\psi(s)$ and $\rho(s)$ become:

$$
\begin{aligned}
& \mathrm{i} \frac{\mathrm{d}}{\mathrm{d} s} \psi(s)=H(s) \psi(s) \\
& \frac{\mathrm{d}}{\mathrm{d} s} \boldsymbol{n}(s)=2 \boldsymbol{h}(s)_{\wedge} \boldsymbol{n}(s) .
\end{aligned}
$$

An easy calculation shows that the most general $H(s)$ has four independent real arbitrary functions of $s$, namely $a(s), b(s), c(s)$ and $d(s)$ in:

$$
\begin{aligned}
& \begin{aligned}
h(s)= & \left(a(s) \cos s, b(s) \cos s, \sqrt{3} c(s)+d(s)\left(\cos ^{2} s-\sin ^{2} s\right),\right. \\
& -a(s) \sin s,-b(s) \sin s, d(s) \cos s \sin s,-1, c(s))
\end{aligned} \\
& h_{0}(s)=\frac{2}{\sqrt{3}} c(s)-d(s) \sin ^{2} s .
\end{aligned}
$$

Moreover we also find with this general Hamiltonian:

$$
\begin{aligned}
\operatorname{Tr}(\rho(s) H(s)) & =(\psi(s), H(s) \psi(s)) \\
& =h_{0}(s)+\frac{2}{\sqrt{3}} \boldsymbol{n}(s) \cdot \boldsymbol{h}(s) \\
& =0 .
\end{aligned}
$$

This is consistent with the vanishing of the dynamical phase as the state evolves along $\{\psi(s)\}$ of equation (4.2), which is directly checked to be true. In addition if we wish to have a constant Hamiltonian producing this evolution, we must set $a(s)=b(s)=d(s)=0$, $c(s)=c_{0}=$ constant and then we get:

$$
H_{\text {constant }}^{(0)}=\left(\frac{2}{\sqrt{3}} \mathbf{1}+\sqrt{3} \lambda_{3}+\lambda_{8}\right) c_{0}-\lambda_{7} .
$$

It is possible to express this simple constant Hamiltonian directly in terms of the end points $\boldsymbol{n}^{(1)}$ and $\boldsymbol{n}^{(2)}$ of the geodesic $C_{\text {geo }}^{(0)}$ given in equation (4.1). Namely one finds:

$$
\left(\boldsymbol{n}_{\wedge}^{(1)} \boldsymbol{n}^{(2)}\right)_{r}=-\frac{3}{2}(\sin \alpha \cos \alpha) \delta_{r 7}
$$

which, if we set the constant $c_{0}$ to zero, leads to:

$$
H_{\mathrm{constant}}^{(0)}=\frac{\boldsymbol{n}_{\wedge}^{(1)} \boldsymbol{n}^{(2)} \cdot \boldsymbol{\lambda}}{\left|\boldsymbol{n}_{\wedge}^{(1)} \boldsymbol{n}^{(2)}\right|} .
$$

We can now generalize these results to any geodesic $C_{\text {geo }} \subset \mathcal{O}$ connecting any two points, $\boldsymbol{n}, \boldsymbol{n}^{\prime} \in \mathcal{O}$. Writing $\boldsymbol{n} \cdot \boldsymbol{n}^{\prime}=\frac{1}{2}\left(3 \cos ^{2} \alpha-1\right)$, we have the result that the constant Hamiltonian

$$
H_{\text {constant }}=\frac{2 \boldsymbol{n}_{\wedge} \boldsymbol{n}^{\prime} \cdot \boldsymbol{\lambda}}{3 \sin \alpha \cos \alpha}
$$

can produce evolution along the geodesic $C_{\text {geo }}$. This generalizes well known results in the case of two-level systems [7]. 


\section{The Pancharatnam geometric phase formula for three-level systems}

The purpose of this section is to obtain the generalization of the result (3.11) for threelevel systems. For this we must determine the geometrical description of the most general geodesic triangle on $\mathcal{O}$, up to an overall $S U$ (3) transformation, and then use the general connection (3.8) to find the geometric phase associated with this triangle.

Let $A, B$ and $C$ be three general points in $\mathcal{O}$ such that no two of them enclose an angle of $\frac{2 \pi}{3}$ radians. Using the freedom of common $S U$ (3) action we can transport $A$ to a position $A^{(0)}$ with a representative vector of the form $\psi^{(1)}$ of equation (4.1). In this process let $B$ and $C$ move to locations $B^{\prime}$ and $C^{\prime}$ :

$$
\begin{aligned}
& A, B, C \stackrel{S U(3)}{\longrightarrow} A^{(0)}, B^{\prime}, C^{\prime}: \\
& A^{(0)} \rightarrow \rho^{(1)}=\psi^{(1)} \psi^{(1) \dagger} \quad \psi^{(1)}=\left(\begin{array}{l}
0 \\
0 \\
1
\end{array}\right) .
\end{aligned}
$$

This $\rho^{(1)}\left(\psi^{(1)}\right)$ is invariant under a $U(2)(S U(2))$ subgroup of $S U(3)$. Exploiting this we can next transport $B^{\prime}$ to a position $B^{(0)}$ while leaving $A^{(0)}$ fixed, and simultaneously $C^{\prime}$ to some $C^{\prime \prime}$, such that:

$$
\begin{aligned}
& A^{(0)}, B^{\prime}, C^{\prime} \stackrel{U(2)}{\longrightarrow} A^{(0)}, B^{(0)}, C^{\prime \prime}: \\
& B^{(0)} \rightarrow \rho^{(2)}=\psi^{(2)} \psi^{(2) \dagger} \quad \psi^{(2)}=\left(\begin{array}{c}
0 \\
\sin \xi \\
\cos \xi
\end{array}\right) \quad 0<\xi<\frac{\pi}{2}
\end{aligned}
$$

thereby introducing the angle $\xi$. We have also secured that $\left(\psi^{(1)}, \psi^{(2)}\right)$ is real positive. Now these two density matrices, $\rho^{(1)}$ and $\rho^{(2)}$, are invariant under a particular diagonal $U(1)$ subgroup of $S U(3)$, whose elements are:

$$
d(\beta)=\operatorname{diag}\left(\mathrm{e}^{-2 \mathrm{i} \beta}, \mathrm{e}^{\mathrm{i} \beta}, \mathrm{e}^{\mathrm{i} \beta}\right) \in S U(3) \quad 0 \leqslant \beta<2 \pi .
$$

We now have the freedom of transformations $d(\beta)$, which leave $A^{(0)}$ and $B^{(0)}$ unchanged, to move $C^{\prime \prime}$ to a convenient position $C^{(0)}$. A little thought shows that this can be achieved as follows:

$$
\begin{aligned}
& A^{(0)}, B^{(0)}, C^{\prime \prime} \stackrel{U(1)}{\longrightarrow} A^{(0)}, B^{(0)}, C^{(0)}: \\
& C^{(0)} \rightarrow \psi^{(3)}=\left(\begin{array}{c}
\sin \eta \cos \zeta \\
\mathrm{e}^{\mathrm{i} \chi_{2}} \sin \eta \sin \zeta \\
\cos \eta
\end{array}\right) \\
& 0<\eta<\frac{\pi}{2} \quad 0 \leqslant \zeta \leqslant \frac{\pi}{2} \quad 0 \leqslant \chi_{2}<2 \pi .
\end{aligned}
$$

We have parametrized $\psi^{(3)}$ in the manner of equation (2.16): the $U(1)$ freedom allows us to transform the phase $\chi_{1}$ to zero, and an overall phase freedom has been used to make $\left(\psi^{(1)}, \psi^{(3)}\right)$ real positive. We now see that the description of the most general geodesic triangle in $\mathcal{O}$, up to an overall $S U$ (3) transformation, involves the four angle parameters $\xi, \eta, \zeta, \chi_{2}$. These are therefore intrinsic to the shape and size of the triangle. This counting agrees with the fact that $S U(3)$ is eight-dimensional, and choosing three points on $\mathcal{O}$ independently involves choosing twelve independent coordinates.

Now we consider the geometric phase for the geodesic triangle $A^{(0)}, B^{(0)}, C^{(0)}$. Using the result (3.8) based on the Bargmann invariant, and the conveniently chosen representative 
vectors in equations (5.1), (5.2), and (5.4), we find:

$$
\begin{aligned}
\varphi_{g}\left[A^{(0)} B^{(0)} C^{(0)}\right] & =-\arg \left(\psi^{(1)}, \psi^{(2)}\right)\left(\psi^{(2)}, \psi^{(3)}\right)\left(\psi^{(3)}, \psi^{(1)}\right) \\
& =+\arg \left(\psi^{(3)}, \psi^{(2)}\right) \\
& =\arg \left(\cos \xi \cos \eta+\sin \xi \sin \eta \sin \zeta \mathrm{e}^{-\mathrm{i} \chi_{2}}\right) \\
& =\arg \left(1+\tan \xi \tan \eta \sin \zeta \mathrm{e}^{-\mathrm{i} \chi_{2}}\right) .
\end{aligned}
$$

This is the generalization of the Pancharatnam formula (3.11). We see that the phase $\chi_{2}$ plays an important role: $\varphi_{g}\left[A^{(0)} B^{(0)} C^{(0)}\right]$ can be nonzero only if $\chi_{2}$ is nonzero.

We can also express this geometric phase directly in terms of the vectors $\boldsymbol{n}^{(1)}, \boldsymbol{n}^{(2)}$ and $\boldsymbol{n}^{(3)} \in \mathcal{O}$ corresponding to the vertices $A^{(0)}, B^{(0)}, C^{(0)}$ of the geodesic triangle:

$$
\begin{aligned}
\varphi_{g}\left[A^{(0)} B^{(0)} C^{(0)}\right] & =-\arg \operatorname{Tr}\left(\rho^{(1)} \rho^{(2)} \rho^{(3)}\right) \\
& =-\tan ^{-1}\left[\frac{2 \sqrt{3} \boldsymbol{n}^{(1)} \cdot \boldsymbol{n}_{\wedge}^{(2)} \boldsymbol{n}^{(3)}}{\left(\boldsymbol{n}^{(1)}+\boldsymbol{n}^{(2)}+\boldsymbol{n}^{(3)}\right)^{2}+2 \boldsymbol{n}^{(1)} \cdot \boldsymbol{n}^{(2)} \star \boldsymbol{n}^{(3)}-2}\right] .
\end{aligned}
$$

In this form the $S U(3)$ invariance is explicit.

We may collect our results of this and the previous section to say: given any geodesic triangle in the ray space $\mathcal{O}$ for three-level systems, it is possible to find a piecewise constant Hamiltonian to produce evolution along this triangle, such that the dynamical phase vanishes, and the geometric phase is then given by the intrinsic expression (5.5) and (5.6).

\section{Concluding remarks}

We have exploited the newly constructed extension of the Poincaré sphere representation from two- to three-level quantum systems, to develop a generalization of the Pancharatnam geometric phase formula for cyclic evolution of such a system along a geodesic triangle in state space. We have found that such a triangle is intrinsically defined by four angle type parameters in contrast to the three needed for defining a triangle on the Poincare sphere, $S^{2}$; and have obtained the explicit and simple expression for the geometric phase in terms of them.

It is easy to check that the original Pancharatnam formula (3.11) emerges from equation (5.5) as a particular case. Namely if we take $\zeta=\frac{\pi}{2}$, the three Hilbert space vectors, $\psi^{(1)}, \psi^{(2)}$ and $\psi^{(3)}$, of equations (5.1), (5.2) and (5.4) all lie in the two-dimensional 2-3 subspace of $\mathcal{H}^{(3)}$, and involve just three angle parameters, $\xi, \eta$ and $\chi_{2}$ :

$$
\psi^{(1)}=\left(\begin{array}{l}
0 \\
0 \\
1
\end{array}\right) \quad \psi^{(2)}=\left(\begin{array}{c}
0 \\
\sin \xi \\
\cos \xi
\end{array}\right) \quad \psi^{(3)}=\left(\begin{array}{c}
0 \\
\mathrm{e}^{\mathrm{i} \chi_{2}} \sin \eta \\
\cos \eta
\end{array}\right) .
$$

Let the corresponding rays be represented by unit vectors $\boldsymbol{n}_{1}, \boldsymbol{n}_{2}$ and $\boldsymbol{n}_{3}$ on an $S^{2}$ and let the sides of the corresponding spherical triangle be $a, b$ and $c$. Then, from equation (2.1), we have:

$$
\begin{aligned}
& \boldsymbol{n}_{1} \cdot \boldsymbol{n}_{2}=\cos a=2\left|\left(\psi^{(1)}, \psi^{(2)}\right)\right|^{2}-1=\cos 2 \xi \\
& \boldsymbol{n}_{1} \cdot \boldsymbol{n}_{3}=\cos b=2\left|\left(\psi^{(1)}, \psi^{(3)}\right)\right|^{2}-1=\cos 2 \eta \\
& \boldsymbol{n}_{2} \cdot \boldsymbol{n}_{3}=\cos c=2\left|\left(\psi^{(2)}, \psi^{(3)}\right)\right|^{2}-1=2\left|\cos \xi \cos \eta+\sin \xi \sin \eta \mathrm{e}^{\mathrm{i} \chi_{2}}\right|^{2}-1 .
\end{aligned}
$$

Thus $a=2 \xi, b=2 \eta$ and in the case of the angle $c$ we have

$$
\cos \frac{c}{2}=\left|\cos \xi \cos \eta+\sin \xi \sin \eta \mathrm{e}^{\mathrm{i} \chi_{2}}\right| \text {. }
$$


Now from equation (5.5) the geometric phase in this case is given by:

$$
\varphi_{g}\left[A^{(0)} B^{(0)} C^{(0)}\right]=\arg \left(\cos \xi \cos \eta+\sin \xi \sin \eta \mathrm{e}^{-\mathrm{i} \chi_{2}}\right)
$$

or equally well (apart from the sign) by:

$$
\cos \left(\varphi_{g}\left[A^{(0)} B^{(0)} C^{(0)}\right]\right)=\frac{\cos \xi \cos \eta+\sin \xi \sin \eta \cos \chi_{2}}{\left|\cos \xi \cos \eta+\sin \xi \sin \eta \mathrm{e}^{\mathrm{i} \chi_{2}}\right|} .
$$

Focusing on the $\chi_{2}$ dependence here (and as we have seen earlier this is the crucial aspect), and using equation (6.2) we have:

$$
\cos \xi \cos \eta+\sin \xi \sin \eta \cos \chi_{2}=\frac{(1+\cos a+\cos b+\cos c)}{4 \cos a / 2 \cos b / 2}
$$

leading to

$$
\cos \left(\varphi_{g}\left[A^{(0)} B^{(0)} C^{(0)}\right]\right)=\frac{(1+\cos a+\cos b+\cos c)}{4 \cos a / 2 \cos b / 2 \cos c / 2} .
$$

However, the right-hand side here is precisely $\cos \frac{1}{2} \triangle(a, b, c)$, where $\triangle(a, b, c)$ is the solid angle subtended at the origin of $S^{2}$ by the spherical triangle with sides $a, b$ and $c$. In this way the Pancharatnam result $\varphi_{g}\left[A^{(0)} B^{(0)} C^{(0)}\right]=\frac{1}{2} \triangle(a, b, c)$ is recovered. (The sign can also be recovered with some additional effort.)

This same verification leads us to the following significant remark: as long as one is interested in cyclic evolution of any quantum system along a geodesic triangle in ray space, however large the dimension of the Hilbert space $\mathcal{H}$ may be, our result (5.5) for the geometric phase is applicable and is completely general. This is because a triangle involves (at most) three independent vectors $\psi^{(1)}, \psi^{(2)}, \psi^{(3)} \in \mathcal{H}$, and these always lie in some three-dimensional subspace of $\mathcal{H}$. Thus, nothing additional is needed to handle geometric phases for cyclic evolutions along geodesic triangles for $N$-level systems, for any $N \geqslant 4$.

Going back to the formula (5.5) based on the standard configuration $\psi^{(1)}, \psi^{(2)}$ and $\psi^{(3)}$ of equations (5.1), (5.2) and (5.4), we see that the geometric phase for a geodesic triangle in a three-level system state space depends on all the four parameters, $\xi, \eta, \zeta$ and $\chi_{2}$, used to describe the triangle. Here the parametrization of the vertices follows the pattern of local coordinates defined for $\mathcal{O}$ in section 2. It is, however, possible to choose another standard configuration for a geodesic triangle such that the geometric phase then depends only on three of the four parameters. Thus by a fixed $S U(3)$ transformation one can arrange to have:

$$
\begin{aligned}
\psi^{(1)^{\prime}}=A \psi^{(1)}=\left(\begin{array}{l}
1 \\
0 \\
0
\end{array}\right) \quad \psi^{(2)^{\prime}}=A \psi^{(2)}=\left(\begin{array}{c}
\cos \xi \\
\sin \xi \\
0
\end{array}\right) \\
\psi^{(3)^{\prime}}=A \psi^{(3)}=\left(\begin{array}{c}
\sin \eta^{\prime} \cos \zeta^{\prime} \\
\mathrm{e}^{\mathrm{i} \chi_{2}} \sin \eta^{\prime} \sin \zeta^{\prime} \\
\cos \eta^{\prime}
\end{array}\right) \quad A=\left(\begin{array}{ccc}
0 & 0 & 1 \\
0 & 1 & 0 \\
-1 & 0 & 0
\end{array}\right) \in S U(3)
\end{aligned}
$$

(where we have retained the form of the local coordinates for $\mathcal{O}$ ), and thus the geodesic triangle is described by four new parameters $\xi, \eta^{\prime}, \zeta^{\prime}$ and $\chi_{2}$ which are functions of $\xi, \eta$, $\zeta$ and $\chi_{2}$. Then the geometric phase becomes

$$
\begin{aligned}
\varphi_{g}\left[A^{(0)^{\prime}} B^{(0)^{\prime}} C^{(0)^{\prime}}\right] & =\varphi_{g}\left[A^{(0)} B^{(0)} C^{(0)}\right] \\
& =-\arg \left(\psi^{(1)^{\prime}}, \psi^{(2)^{\prime}}\right)\left(\psi^{(2)^{\prime}}, \psi^{(3)^{\prime}}\right)\left(\psi^{(3)^{\prime}}, \psi^{(1)^{\prime}}\right) \\
& =+\arg \left(\psi^{(3)^{\prime}}, \psi^{(2)^{\prime}}\right) \\
& =\arg \left(1+\tan \xi \tan \zeta^{\prime} \mathrm{e}^{-\mathrm{i} \chi_{2}}\right) .
\end{aligned}
$$


Now there is no dependence on $\eta^{\prime}$, and this has happened because $\tan \eta \sin \zeta=\tan \zeta^{\prime}$. The expression (6.9) in fact coincides in structure with what one would have obtained for a two-level system, i.e. the original Pancharatnam result (6.4) with suitable reinterpretation of parameters. The explanation for this is that in the expression for the three-vertex Bargmann invariant involving vectors $\psi^{(1)}, \psi^{(2)}$ and $\psi^{(3)}$ only the projection of $\psi^{(3)}$ onto the twodimensional subspace spanned by $\psi^{(1)}$ and $\psi^{(2)}$ is relevant.

A discussion of feasible experimental schemes to check the validity of our main result (5.5), at least in some nontrivial cases which do go beyond the two-level situation, will be the subject of a forthcoming publication.

\section{Acknowledgments}

We thank Rajaram Nityananda and Joseph Samuel for useful comments. Arvind thanks the University Grants Commission, India, for financial support and KSM thanks the JNCASR for a Visiting Fellowship and the CTS for providing facilities.

\section{References}

[1] Berry M V 1984 Proc. R. Soc. A 39245

[2] Ramaseshan S and Nityananda R 1986 Curr. Sci. 551225

[3] Pancharatnam S 1956 Proc. Ind. Acad. Sci. A 44247

[4] Berry M V 1987 J. Mod. Opt. 341401

[5] Mukunda N and Simon R 1993 Ann. Phys., NY 228205

[6] Born M and Wolf E 1987 Principles of Optics (New York: Pergamon)

Ramachandran G N and Ramaseshan S 1961 Crystal optics Handbuch der Physik vol XXV/1 (Berlin: Springer)

See also Simon R, Mukunda N and Sudarshan E C G 1989 Pramana J. Phys. 32769

[7] See for instance Simon R, Mukunda N and Sudarshan E C G 1989 Pramana J. Phys. 32769

Simon R and Mukunda N 1992 J. Phys. A: Math. Gen. 256131

[8] Samuel J and Bhandari R 1988 Phys. Rev. Lett. 602339

See also [5] above

[9] Bargmann V 1964 J. Math. Phys. 5862

See also [5] above

[10] Khanna G, Mukhopadhyay S, Simon R and Mukunda N 1997 Geometric phases for SU(3) representations and three level quantum systems Ann. Phys., $N Y$ in press

Mukunda N 1996 Three level quantum systems and SU(3) geometric phases Preprint IISc

[11] Bouchiat C and Gibbons G W 1988 J. Physique 49187

[12] Poincaré H (ed) 1892 Theorie Mathematique de la Lumiere (Paris: George Carre) p 275

[13] Gell-Mann M and Neeman Y 1964 The Eightfold Way (New York: Benjamin) de Swart J J 1963 Rev. Mod. Phys. 35916 